\title{
Resolution of Diabetes Insipidus After Pyeloplasty: A Case Report and Review of the
} Literature

\author{
Christina P. Carpenter ${ }^{1}$, Ashley Rawson ${ }^{2}$, David S. Hains ${ }^{2}$, Dana W. Giel ${ }^{1}$ \\ ${ }^{1}$ Division of Pediatric Urology \\ Department of Urology \\ Le Bonheur Children's Hospital \\ The University of Tennessee Health Science Center \\ Memphis, TN
}

${ }^{2}$ Division of Nephrology

Department of Pediatrics

Riley Hospital for Children

Indiana University School of Medicine

Indianapolis, IN

Key Words: Diabetes insipidus, pyeloplasty, ureteropelvic junction obstruction, pediatrics

Acknowledgements: None

\section{Corresponding Author:}

Christina Carpenter, MD

Le Bonheur Children's Hospital

Division of Pediatric Urology

49 North Dunlap Street, Suite 305

Memphis, TN 38105

Office: (901) 287-7473

Email: carpenter.christina@gmail.com

This is the author's manuscript of the article published in final edited form as:

Carpenter, C. P., Rawson, A., Hains, D. S., \& Giel, D. W. (2018). Resolution of Diabetes Insipidus after Pyeloplasty: 


\begin{abstract}
Nephrogenic diabetes insipidus (NDI), a rare cause of polyuria and polydipsia in children, is usually managed with medications and careful monitoring of water intake. We present a child who was incidentally found to have right hydronephrosis secondary to ureteropelvic junction obstruction, and was subsequently also diagnosed with NDI. After being medically managed, he underwent open right pyeloplasty. His polydipsia abated within one month of surgery, and he has done well off of medications since that time. NDI resolution after correction of obstructive uropathy in adults has been reported, but this represents a novel case in pediatrics.
\end{abstract}

Abbreviations: Ureteropelvic junction obstruction (UPJO), nephrogenic diabetes insipidus (NDI), acute kidney injury (AKI), vasopressin V2 receptor (AVPR2), aquaporin $(\mathrm{AQP})$

\title{
Introduction
}

Nephrogenic diabetes insipidus (NDI) is a rare cause of polyuria and polydipsia in children. Its diagnosis requires dilute urine in the presence of elevated serum plasma osmolality [1]. The majority of cases are inherited, but the condition can be acquired as a result of medications, biochemical abnormalities, and/or obstructive uropathy [2]. This latter etiology incites the disease by damaging principal cells of the cortical collecting 
duct, thus rendering them unresponsive to antidiuretic hormone [3]. NDI is very rare in pediatric patients, and is described mostly in children with congenital obstructions of the urinary tract. We present a case in which NDI secondary to ureteropelvic junction obstruction (UPJO) resolved completely after pyeloplasty. Resolution of NDI due to obstructive uropathy has been reported in adults [4-6]. NDI's resolution after surgery, however, is novel in pediatric patients.

\section{Case Presentation}

A 19-month-old boy was brought in by Emergency Medical Services after being found lethargic, febrile, and suffering from respiratory distress at home. He was intubated in the field prior to transport to Le Bonheur Children's Hospital. Upon arrival, his creatinine was found to be $0.8 \mathrm{mg} / \mathrm{dl}$, and his transaminases were markedly elevated. Due to his clinical status and the suspicion of neglect and abuse, a CT was performed, which demonstrated right hydronephrosis tapering to a narrowed right ureter, suggestive of UPJO (Figure 1). No evidence of concomitant infection was present.

Urology was consulted, and a MAG3 nuclear renogram with furosemide was performed. This study showed approximately equal distribution of renal function, and confirmed the suspected right-sided obstruction. With intravenous hydration over the next few days, his multiorgan dysfunction completely resolved. His creatinine peaked 
at $1.5 \mathrm{mg} / \mathrm{dl}$, but then trended down to $0.3 \mathrm{mg} / \mathrm{dl}$. Urology follow-up was coordinated in order to allow him to recover from his acute illness before proceeding with pyeloplasty.

Nephrology was also consulted during his hospitalization due to his electrolyte abnormalities and acute kidney injury (AKI). On admission, his serum sodium and osmolality were elevated (158 mmol/L and $337 \mathrm{mOsm} / \mathrm{kg}$, respectively), but his urine was dilute (342 mOsm/kg). His hypernatremia normalized over his hospital course, and was believed to be secondary to hypovolemia and/or AKI. At his follow-up appointment one month after discharge, however, his creatinine had increased $(0.7$ $\mathrm{mg} / \mathrm{dl}$ ), and his foster family noted that he would drink from the toilet if water was not freely available. This clinical picture raised the concern for NDI. His caretakers were counseled on increasing his water intake and on monitoring the color of his urine as a measure for adequate hydration. He was also started on amiloride (5mg three times per day) and hydrochlorothiazide (12.5mg three times per day). At his next visit two months later, his caretakers noted that his polyuria and polydipsia were markedly improved.

Four months after his initial diagnosis, he was admitted for intravenous hydration in preparation for surgery. His serum and urine osmolalities at that time were $298 \mathrm{mOsm} / \mathrm{kg}$ and $245 \mathrm{mOsm} / \mathrm{kg}$, respectively. The following day, he underwent right pyeloplasty, and was discharged uneventfully on the first postoperative day. Of 
note, the delay in intervention was due to social issues with follow-up and optimal medical management of his NDI.

One month postoperatively, his foster parents noted that his polydipsia had completely resolved. His medications were discontinued, and his caretakers were instructed to contact the clinic if he resumed his previous behaviors. Since that time, his ureteral stent has been removed, and he has had no recurrence of his NDI symptoms. Labs from his most recent visit confirm resolution with respective serum and urine osmolalities of $299 \mathrm{mOsm} / \mathrm{kg}$ and $740 \mathrm{mOsm} / \mathrm{kg}$.

\section{Discussion}

Diabetes insipidus has a prevalence of 1 in 25,000 children. An estimated 10\% of these cases are nephrogenic [7], which occurs when there is a disruption of reabsorption of water by the principal cells lining the collecting duct [2]. Normally, this process is initiated by the binding of vasopressin to the vasopressin V2 receptors (AVPR2) on these cells' membranes. This interaction stimulates fusion of intracellular aquaporin-2 (AQP2)-containing vesicles with the apical membranes. Then, in conjunction with AQP3 and AQP4 on the basolateral sides, water reabsorption occurs [2,10].

Several researchers have attempted to explain the mechanism by which obstructive uropathy causes NDI. Frokiaer et al demonstrated that obstruction decreases transcription of the $A Q P 2$ gene, thus decreasing the reabsorption abilities of the 
principal cells [8]. Furthermore, Hong et al suggested that the increased hydrostatic pressure in the collecting ducts damages the tubular epithelium, thus decreasing sensitivity of the AVPR2 receptors to vasopressin [5]. Interestingly, Frokiaer et al have also shown that in unilateral obstruction, as was seen in our patient, the contralateral kidney too demonstrates decreased AQP2 expression. They believe this to be due to a renorenal mechanism compensating for the impaired excretion of the affected side [9].

Reports in adult literature have demonstrated that release of obstruction can correct the defect. Hong et al presented a patient who was cured of his symptoms after surgical resection of his enlarged prostate [5], and Kato et al described a man whose urine output returned to normal without medications six months after nephrostomy tube decompression of his hydroureteronephrosis, which was caused by ileal leiomyosarcoma [6]. Yoshiokoa et al report a patient with a similar result after drainage of his obstructed solitary kidney [4]. Not all patients, however, are cured with resolution of obstruction. Gungor et al present a patient with bilateral hydroureteronephrosis due to a large ovarian mass. She, unfortunately, continued to require thiazides even after pelvic exenteration and relief of her ureteral obstruction [10].

Acquired NDI in children is usually reported in the setting of congenital abnormalities, such as posterior urethral valves or other outlet obstruction [11,12]. In these patients, the severe impediment to urinary drainage impaired in utero renal 
development. Thus, even after optimization of their urinary tract surgically, they continue to require medications and careful monitoring due to persistent symptoms. Our patient's NDI, however, resolved completely. This demonstrates a novel case of renal function salvage with surgical correction.

Authors' Note: Consent was obtained from patient's legal guardian for submission and publication of this case study. 


\section{References}

[1] Yuksel OH, Kivrak M, Sahin A, Akan S, Urkmez A, Verit A. A case of idiopathic diabetes insipidus presented with bilateral hydroureteronephrosis and neurogenic bladder: A pediatric case report and literature review. Can Urol Assoc J 2015;9:817.

[2] Bockenhauer D, Bichet DG. Pathophysiology, diagnosis and management of nephrogenic diabetes insipidus. Nat Rev Nephrol 2015;11:576-88.

[3] Saborio P, Tipton GA, Chan JCM. Diabetes Insipidus. Pediatr Rev 2000;21:122-9.

[4] Yoshioka K, Imanishi M, Sakai H, Morikawa T, Okada N, Konishi Y, et al. Nephrogenic diabetes insipidus due to hydronephrosis in a patient with a solitary kidney. Clin Exp Nephrol 2003;7:243-6.

[5] Hong E-G, Suh Y, Chung Y-S, Kim H-M, Shin G-T, Chung D-Y, et al. A Case of Nephrogenic Diabetes Insipidus Caused by Obstructive Uropathy Due to Prostate Cancer. Yonsei Med J 2000;41:150-4.

[6] Kato A, Hishida A, Ishibashi R, Nakajima T, Ohura M, Furuya R, et al. Nephrogenic Diabetes Insipidus Associated with Bilateral Ureteral Obstruction. Intern Med 1994;33:231-3.

[7] Dabrowski E, Kadakia R, Zimmerman D. Diabetes insipidus in infants and children. Best Pract Res Clin Endocrinol Metab 2016;30:317-28.

[8] Frokiaer J, Marples D, Knepper MA, Nielsen S. Bilateral ureteral obstruction downregulates expression of vasopressin-sensitive AQP-2 water channel in rat kidney. Am J Physiol-Ren Physiol 1996;270:F657-F668.

[9] Frokiaer J, Christensen BM, Marples D, Djurhuus JC, Jensen UB, Knepper MA, et al. Downregulation of aquaporin-2 parallels changes in renal water excretion in unilateral ureteral obstruction. Am J Physiol-Ren Physiol 1997;273:F213-F223.

[10] Gungor T, Kokanalý MK, Öztürkkan D, Tapisiz OL, Mollamahmutoglu L. A case of nephrogenic diabetes insipidus caused by partial bilateral ureteral obstruction due to advanced stage ovarian carcinoma. Arch Gynecol Obstet 2009;280:679-81.

[11] Becker A, Baum M. Obstructive uropathy. Early Hum Dev 2006;82:15-22.

[12] Baum NH, Burger R, Carlton CE. Nephrogenic diabetes insipidus: Associated with posterior urethral valves. Urology 1974;4:581-583. 
Figure 1. CT scan demonstrating right hydronephrosis secondary to UPJO.

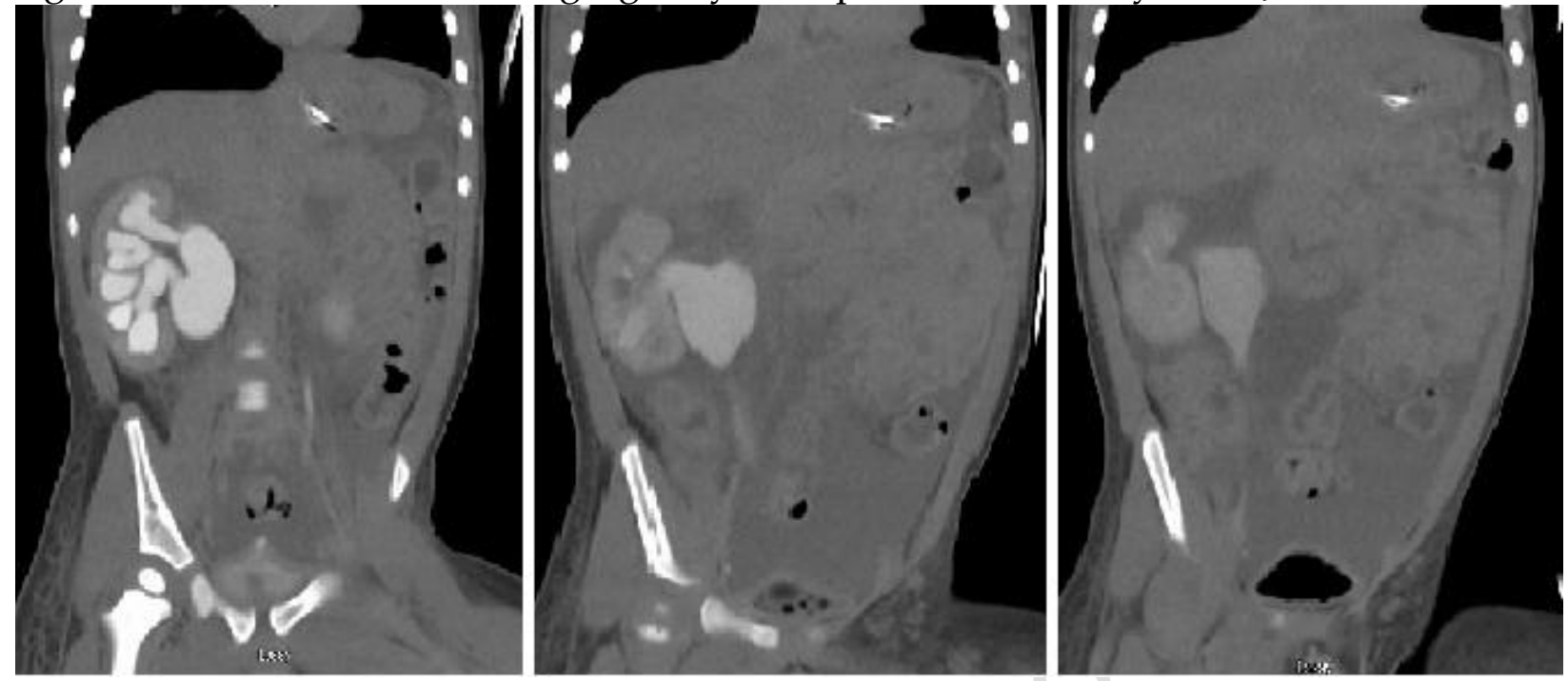

